\title{
Nanofibrillation of pulp fibers by twin-screw extrusion
}

\author{
Thao Thi Thu Ho Kentaro Abe • \\ Tanja Zimmermann · Hiroyuki Yano
}

Received: 17 July 2014 / Accepted: 26 November 2014/Published online: 14 December 2014

(C) Springer Science+Business Media Dordrecht 2014

\begin{abstract}
The effect of the fibrillation process through a twin-screw extruder (TSE) on properties of pulp fibers was studied, considering the degree of both fibrillation and degradation of the fibers. Neverdried refined bleached kraft pulp (NBKP) was passed through a TSE several times at a high concentration of $28 \mathrm{wt} \%$. The output of fibrillated fibers had a solid content up to ca. $50 \mathrm{wt} \%$, and the material was in powder form. Characterizations of the morphology, dewatering speed, sedimentation, laser light scattering, scanning electron microscopy of cellulose suspensions, and light transmittance of resin-impregnated films showed that the fibrillation degree of the pulp was enhanced with a higher number of passes. However, the results from thermogravimetry, intrinsic viscosity, and X-ray diffraction analyses indicated that some degradation occurred during the fibrillation process in the TSE. In addition, the mechanical properties of the fibrillated pulp sheets reflected the effects of treatment on the fibrillation and degradation of the cellulose. For never-dried refined NBKP pulp, the best compromise in terms of fibrillation and
\end{abstract}

T. T. T. Ho $\cdot$ K. Abe $\cdot$ H. Yano

Research Institute for Sustainable Humanosphere, Kyoto University, Kyoto, Japan

T. T. T. Ho $(\bowtie) \cdot$ T. Zimmermann

Applied Wood Materials, Swiss Federal Laboratories for

Materials Science and Technology, Duebendorf,

Switzerland

e-mail: cohothu@gmail.com degradation degree is between 3 and 14 passes, depending on the envisaged properties and applications. The possibility of nanocellulose production at the reported high solid contents is of great interest for industry.

Keywords Nanofibrillated cellulose $\cdot$ Twin-screw extruder - Degree of fibrillation - Degree of degradation

\section{Introduction}

Nanocellulose is an interesting renewable material for many industrial applications because of its excellent property profile (Dufresne 2012). Different terms are used in the literature for the high aspect ratio of cellulose nanofibers such as microfibrillated cellulose or cellulose nanofibrils. In the following text, the term nanofibrillated cellulose (NFC) will be used. NFC has been isolated from different raw materials such as wood, agricultural waste, or even recycled newspaper (Josset et al. 2014; Siró and Plackett 2010). The fibrillation of cellulose fibers into NFC is generally done by mechanical, chemical, or enzymatic treatments and combinations thereof (Henriksson et al. 2007, 2008; Hubbe et al. 2008; Iwamoto et al. 2007; Paakko et al. 2007; Zimmermann et al. 2005, 2010).

In processing such as mixing, blending, compounding, or reacting of thermoplastic polymers, twin screw 
extruders have been extensively used. Twin-screw extruders (TSEs) offer the processing of polyethylene (PE), polypropylene (PP), polyethylene terephthalate (PET), polyvinyl chloride (PVC), or other polymers (Arthur and Anthony 1998; White 1991). On the contrary, TSE has not been solely considered for the fibrillation of cellulose.

In the context of the pretreatment of biomass for the production of fermentable sugars, an extrusion process has been applied. The alkaline twin-screw extrusion pretreatment was found to improve the enzymatic hydrolysis efficiency of the biomass (Lin et al. 2012; Liu et al. 2013). In addition, the application of additives such as ethylene glycol as well as hotcompressed water treatment enhanced the fibrillation of woody biomass in the TSE (Lee et al. 2009a, 2010).

To a certain extent, the use of a TSE for disintegration of wood has been described in earlier work. Wood fibers of high aspect ratio were separated from wood chips during the twin-screw extrusion process (Hietala et al. 2011a). The pretreatment of wood chips with sodium sulfite enhanced the disintegration process and hence increased the mechanical properties of the wood-polymer composites (Hietala et al. 2011b). The nanofibrillation of bleached wood fibers or TEMPO-oxidized cellulose fibers, however, was not achieved when compounding of nanocomposites of thermoplastic starch with $10 \mathrm{wt} \%$ of fibers in a TSE. In this extrusion condition, low viscosity of the starchfiber mixtures resulting from the high water content of the used premixes $(74 \mathrm{wt} \%$ ) could be the explanations for not obtaining NFC (Hietala et al. 2014). In a similar way, disintegration of cellulose fibers using a TSE has been demonstrated since 2003 and was published in 2007 (Yano et al. 2007) in Japan. It has been used in the works of Suzuki et al. $(2013,2014)$ where the fibrillation actually happened during mixing of pulp fibers with powdered polypropylene (PP) in a TSE.

At any rate, systematic evaluation of the disintegration as well as degradation of cellulose fibers in a TSE has not been carried out previously.

Disintegration of cellulose fibers with a TSE has several advantages compared with other fibrillation methods such as high-shear homogenization or grinding. First, a remarkably high solid content, i.e., a 25-40 wt\% fiber suspension, can be processed through the extruder. The output of fibrillated fibers even has a solid content up to ca. $50 \mathrm{wt} \%$. This is remarkable as with other homogenization methods the optimal solid content of fibrillated material is about $2 \mathrm{wt} \%$ (Dufresne 2013). The fibrillation process through a TSE could therefore result in low production costs due to the high efficiency and fast procedure. Second, the nanocellulose obtained from an extruder is in solid state (powder form instead of an aqueous paste or suspension). Compared to fibrillated cellulose aqueous suspensions, the solid fibrillated cellulose powder brings a great advantage for industries, considering transportation, production, and storage (storage volume and storage life) issues. Third, as TSEs are traditionally used for polymers and composites, the availability and popularity of TSEs is much higher than for other disintegration or homogenization systems such as high-shear homogenizers (e.g., Microfluidizers ${ }^{\circledR}$ ). Therefore, an extruding process for cellulose pulp would be very attractive for industries.

However, one should also take into account some issues when using a TSE as a fibrillation method for pulp fibers. The cellulose obtained from a TSE needs to be re-dispersible in solvents in order to be used in further industrial applications. This could be a great challenge as at high solid contents, the NFC can form irreversible agglomerations called hornification (Eyholzer et al. 2010; Hult et al. 2001). In addition, during the processing by a TSE, degradation of cellulose fibers could occur. This might be due to kneading and breaking mechanisms, the friction between pulp fibers, the increase in temperature during extrusion processing, and the high solid content of the input pulp.

This study aimed at a thorough characterization and quality assessment of NFC produced by twin-screw extrusion. The fibrillated cellulose fibers were obtained by passing never-dried refined NBKP through a TSE $1-14$ times. The fibers were collected after 1, 3, 5, 10, and 14 passes, respectively. The fibers were then re-dispersed in water and characterized using various methods. The obtained materials were compared in terms of the degree of fibrillation and the degradation of cellulose upon treatment.

\section{Experimental}

\section{Materials}

Never-dried refined NBKP (needle-leaf bleached kraft pulp) supplied by Oji Holdings Corp. (Tokyo, Japan) 
with a solid content of $23.9 \mathrm{wt} \%$ was used as raw material. The pulp contains ca. $80 \mathrm{wt} \%$ cellulose and $20 \mathrm{wt} \%$ hemicellulose. This eight-pass refined pulp has a freeness number (Canadian Standard Freeness, CSF) $<10 \mathrm{ml}$ as determined according to TAPPI standard T227om-09.

Fibrillation process with a twin-screw extruder

Prior to processing in the TSE, the solid content of never-dried refined NBKP was adjusted to ca. $28 \mathrm{wt} \%$ by pressing out the excess water in the pulp. The single never-dried sheets of pulp were torn into pieces of ca. $1 \mathrm{~cm} \times 1 \mathrm{~cm}$ and then fed into the TSE [TSE, KZW15TW-30/45MG-NH(-2200), Technovel Corp., Osaka, Japan]. The length to diameter ratio (L/D) of the TSE was 45. The TSE screw was a combination of kneading and feeding screws. The two-tip rotor-type screw segment configuration was selected as the outcome of preliminary experiments. This type of screw fibrillated wet pulp effectively. The operating temperature of the TSE was set to $0{ }^{\circ} \mathrm{C}$. This temperature was controlled to be as close to $0{ }^{\circ} \mathrm{C}$ as possible, and the kneading part of the extruder never exceeded $40{ }^{\circ} \mathrm{C}$ by circulating antifreeze liquid $\left(-5{ }^{\circ} \mathrm{C}\right)$. Without such a cooling system, the temperature of pulp increases above 70 or $80{ }^{\circ} \mathrm{C}$, which allows evaporation of the moisture in pulp and therefore drying of the pulp. The screw speed was $400 \mathrm{rpm}$.

The pulp material was kneaded 1, 3, 5, 10, and 14 passes through the TSE. It was not possible to process the material for more than 14 passes since the temperature of the extruder then exceeded $40{ }^{\circ} \mathrm{C}$. By increasing the number of passes, defibrillation still proceeded; however, the cellulose fibers became discolored because of heat generation. The fibrillated material was obtained as a humid powder with varying solid contents from 32.75 ( 1 pass), 33.39 (3 passes), 36.10 (5 passes), 39.19 (10 passes), to $44.80 \mathrm{wt} \%$ (14 passes). To disperse this kneaded powder in water for further characterization, it was diluted into an aqueous cellulose suspension of ca. $1 \mathrm{wt} \%$ and afterwards homogenized three times with an Ultra-Turrax, each time for $5 \mathrm{~min}$. The process with the Ultra-Turrax (Ultra-Turrax T25 digital, IKA) was initially checked to have no effect on further fibrillation: while the morphology and particle size of fibrillated fibers from the Ultra-Turrax and magnetic stirring dispersion processes were similar, the dispersion process by magnetic stirring took much longer, i.e., 7 days. Therefore, fibrillated cellulose dispersion by using an Ultra-Turrax was preferred in this study.

\section{Characterization}

\section{Morphology}

The morphology of pulp fibers before and after fibrillation was investigated with a field emission scanning electron microscope (FE-SEM, JSM-6700F; JEOL, Tokyo, Japan). A drop of the Ultra-Turraxtreated aqueous cellulose suspension was further diluted using ca. $20 \mathrm{ml}$ of ethanol in a Teflon petri disk. The suspension was spread well in the petri disk before being dried in the oven at $110{ }^{\circ} \mathrm{C}$. After a thin, dried, low-density film had formed on the petri disk, a small area of the film was cut out and glued on a piece of carbon adhesive tape fixed on a specimen holder. The sample was sputter-coated with a platinum (Pt) layer of about 2 nm (JFC-1600; JEOL, Tokyo, Japan). SEM images were recorded with an accelerating voltage of $1.5 \mathrm{kV}$ and at a working distance of $8 \mathrm{~mm}$.

\section{Particle size distribution}

The relative changes of particle size distribution were evaluated by the laser light scattering method using a particle size distribution analyzer (LA-950V2, HORIBA). Some drops of $1 \mathrm{wt} \%$ aqueous cellulose suspensions were diluted in a 280-ml built-in sample bath containing distilled water and then 3-min sonicated for homogeneous dispersion. Each suspension was measured five times.

\section{Light transmittance}

The low-density films of all cellulose materials (0-14 passes through the TSE) were prepared for light transmittance measurements. The term "low density" was used as these films were prepared with ethanol instead of water. $100 \mathrm{ml}$ of aqueous cellulose suspensions of 0.2 wt $\%$ was magnetically stirred overnight. The suspensions were vacuum-filtered using a polytetrafluoroethylene membrane filter with $0.1-\mu \mathrm{m}$ pore size and a 300-mesh metallic sieve. The obtained wet sheets were then immersed in ethanol overnight. The sheets were hot-pressed with a load of $0.1-0.2 \mathrm{kgf} /$ $\mathrm{cm}^{2}$ for $5 \mathrm{~min}$ and then for $15 \mathrm{~min}$ under $3.0 \mathrm{kgf} / \mathrm{cm}^{2}$ 
(the loading was slowly increased in mounting steps). The hot-pressing temperature was $120^{\circ} \mathrm{C}$.

The low-density hot-pressed films were cut into small pieces of $2 \mathrm{~cm} \times 2 \mathrm{~cm}$ and were subsequently immersed in acrylic resin under vacuum for $24 \mathrm{~h}$. The acrylic resin was prepared by mixing acetylaminobenzene propenoic ester ABPE-10 (Mitsubishi Chemical Corp., refractive index: 1.536 ) with $2-3 \mathrm{wt} \%$ of 2-hydroxy-2-methylpropiophenone as curing agent. After being taken out of the resin, the films were sandwiched between micro slide glasses (S9213, Matsunami Glass, Japan) and cured with UV light (20 J/cm², a F300S UV lamp system and a LC6 benchtop conveyer, Fusion UV Systems, Japan). Immediately after curing, the composite films were removed from the glasses and their transparency measured.

The regular light transmittances of low-density hot-pressed films impregnated with acrylic resin were measured at wavelengths from 200 to $800 \mathrm{~nm}$ using an ultraviolet-visible (UV-Vis) spectrometer with a 60 -mm-diameter integrating sphere (U-4100, Hitachi High-Tech Corp., Japan). Regular transmittance was measured by placing the specimens $25 \mathrm{~cm}$ from the entrance port of the integrating sphere. Five specimens were used to characterize each composite film. The thickness of the films was measured at several points, and the average values were taken. The regular light transmittances of the films were normalized for a $50-\mu \mathrm{m}$-thick film. This can be done by converting transmittance values into absorbance values before normalizing the absorbance by the thickness of the film according to Beer's law. The normalized absorbance was then converted back to the transmittance value (Portnoy 1998). These normalized transmittance values and their average were determined.

\section{Dewatering speed}

A specially designed vacuum filter with two hollow cylinders was used in dewatering speed tests. In between these cylinders, a filtration system was set up composed of a mechanical stable sieve of large pore size and filter papers. Three sheets of quantitative ashless filter paper with a diameter of $185 \mathrm{~mm}$ (5A, Advantec Toyo Kaisha Ltd., Japan) were wetted with distilled water prior to the test. Filter papers were sandwiched between O-rings and placed on the sieve before fixing the upper cylinder. A vacuum of $-30.3 \mathrm{kPa}( \pm 0.4)$ was applied briefly while closing the lock between the two cylinders. Then $600 \mathrm{~g}$ of aqueous cellulose suspension of $0.1 \mathrm{wt} \%$ at $20^{\circ} \mathrm{C}$ was poured into the upper cylinder. The suspension was then gently stirred before applying a vacuum of $-30.3 \mathrm{kPa}( \pm 0.4)$ to the lower cylinder. The filtration time through the system for all fibrillated materials as well as the refined pulp was recorded.

\section{Sedimentation speed}

Fifty milliliters of all aqueous cellulose suspensions with a solid content of $0.2 \mathrm{wt} \%$ was transferred to transparent bottles. The bottles were sealed and then shaken 30 times upside-down before being left to stabilize. Pictures were taken immediately and then again after 24 h, 1 week, and 2 weeks, respectively. The sedimentation of the suspensions was qualitatively evaluated based on the photographs.

\section{Thermal degradation}

The sheets of all fibrillated cellulose materials as well as the non-treated NBKP were prepared for thermogravimetric analyses, X-ray diffraction (XRD) measurements, and tensile tests. Different from the lowdensity films (see "Light transmittance" section), these all-cellulose sheets were prepared with water only, which means the immersions of the sheets in ethanol and acrylic resin were skipped.

The pyrolytic behaviors of hot-pressed sheets were analyzed for all the sheets produced using a thermogravimetric analyzer (Q50, TA Instruments, New Castle, DE, USA). Samples with a weight of about $5 \mathrm{mg}$ were heated to $600{ }^{\circ} \mathrm{C}$ with a heating rate of $10{ }^{\circ} \mathrm{C} / \mathrm{min}$ under a dried $\mathrm{N}_{2}$ atmosphere $(60 \mathrm{ml} / \mathrm{min})$. The apparatus recorded the mass loss as a function of temperature.

\section{Degree of polymerization (DP)}

All cellulose materials received after processing in the TSE as well as the starting refined pulp were dried at $50{ }^{\circ} \mathrm{C}$ in an oven for $24 \mathrm{~h}$. A defined amount of cellulose material $(\mathrm{m})$ was dissolved in cupriethylenediamine (CED) solution. The flow time $(t)$ of this mixture through a marked distance of a capillary-tube viscometer was recorded. The intrinsic viscosity was 
calculated based on $m$ and $t$ parameters according to ISO 5351 (2004), which has been used previously for the calculation of the degree of polymerization (DP) (Zimmermann et al. 2010). The intrinsic viscosity, [ $\eta$ ] $(\mathrm{ml} / \mathrm{g})$, is related to DP via the Staudinger-MarkHouwink equation:

$[\eta]=K * D P^{a}$

where the constants $K$ and $a$ depend on the polymersolvent system. For cellulose dissolved in CED solution, two different regimes were found because of formation of superstructures at a DP of ca. 950 (Gruber and Gruber 1981). Accordingly, $K=2.28$ and $a=0.76$ were defined for DP above ca. 950 and $K=0.42$ and $a=1$ for DP below ca. 950 .

\section{Crystallinity}

$\mathrm{X}$-ray diffraction measurements were carried out with an X-ray diffractometer (UltraX 18HF, Rigaku Corp., Tokyo, Japan) with $\mathrm{CuK} \alpha$ radiation $(40 \mathrm{kV}$ and $300 \mathrm{~mA}$ ) from $5^{\circ}$ to $40^{\circ}$ in reflection mode. The samples for XRD were prepared by hot-pressing the fibrillated cellulose nanofibers as well as the non-fibrillated material at $120{ }^{\circ} \mathrm{C}$ into dried sheets (see "Thermal degradation”). Crystallinity (CR) was calculated (Ho et al. 2011; Segal et al. 1959; Thygesen et al. 2005):

$\mathrm{CR}=1-\frac{I_{1}}{I_{2}}$

where $I_{1}$ is the intensity of diffraction at the minimum between $2 \theta=18^{\circ}$ and $19^{\circ}$ and $I_{2}$ the intensity of the crystalline peak at the maximum between $2 \theta=22^{\circ}$ and $23^{\circ}$.

\section{Mechanical properties}

Test specimens of 30-mm length and 6-mm width were cut from all hot-pressed all-cellulose sheets (see "Thermal degradation"). These specimens were conditioned at $50 \% \mathrm{RH}$ and $20{ }^{\circ} \mathrm{C}$. Tensile tests of all sheets were performed with a universal materials testing machine (Model 3365, Instron Corp., Norwood, MA, USA) at a crosshead speed of $10 \mathrm{~mm} / \mathrm{min}$ with a gauge length of $20 \mathrm{~mm}$. The average thickness of the films was measured at four points. The average values of the E-modulus, tensile strength and strain at break were calculated from five specimens. All the measurements were performed at $20{ }^{\circ} \mathrm{C}$ and $50 \% \mathrm{RH}$.

\section{Results and discussion}

Effect of number of passes through a TSE on the degree of fibrillation of cellulose pulp fibers

The degree of fibrillation of cellulose fibers was evaluated by scanning electron microscopy (SEM), dewatering speed, light transmittance of resin-impregnated films, laser light scattering, and sedimentation tests.

SEM images of the starting material and fibrillated cellulose fibers after varying passes through a TSE are displayed in Fig. 1. The images show that the extruding process has a strong effect on the fibrillation of the cellulose fibers. The starting material is to a certain extent already surface fibrillated (Fig. 1a), which is caused by the refining steps during production of starting material NBKP. After passes 1 through 3 (Fig. 1b, c), micro-sized fibers are still visible. Bigger fibril aggregates even exist after five passes (Fig. 1d); however, they are getting smaller, and the cellulose material is more homogeneous after reaching 10 passes (Fig. 1e) or 14 passes (Fig. 1f). The change in the morphology of the fibers is, however, not significant between fibers after 10 passes and 14 passes. In general, the higher the number of passes, the higher the fibrillation effect. This behavior is comparable to other disintegration methods such as high-shear homogenization (Zimmermann et al. 2010) or grinding where the most homogeneous material (with and without pretreatment) is also obtained after approximately ten passes (Iwamoto et al. 2005; Josset et al. 2014). Regarding the morphology, the fibrillated cellulose fibers treated with a TSE appear quite similar compared to fibers passed through a grinder (Iwamoto et al. 2007). This means the fibrillated fibers are highly entangled and twisted together; therefore, it is almost impossible to observe a single long fibril in SEM images. Such fibril morphology could therefore be assessed as a typical result of the grinding or kneading mechanism.

Figure 2 shows the particle size of cellulose fibers after consecutive passes through the TSE. The size distinctly decreased during the first 3 passes and then leveled off with higher numbers of passes, i.e., 10 and 15 passes, respectively. These results indicate that the fibrillation of cellulose fibers was accomplished by ten passes through the TSE. It agrees very well with SEM analysis part discussed above where no considerable 

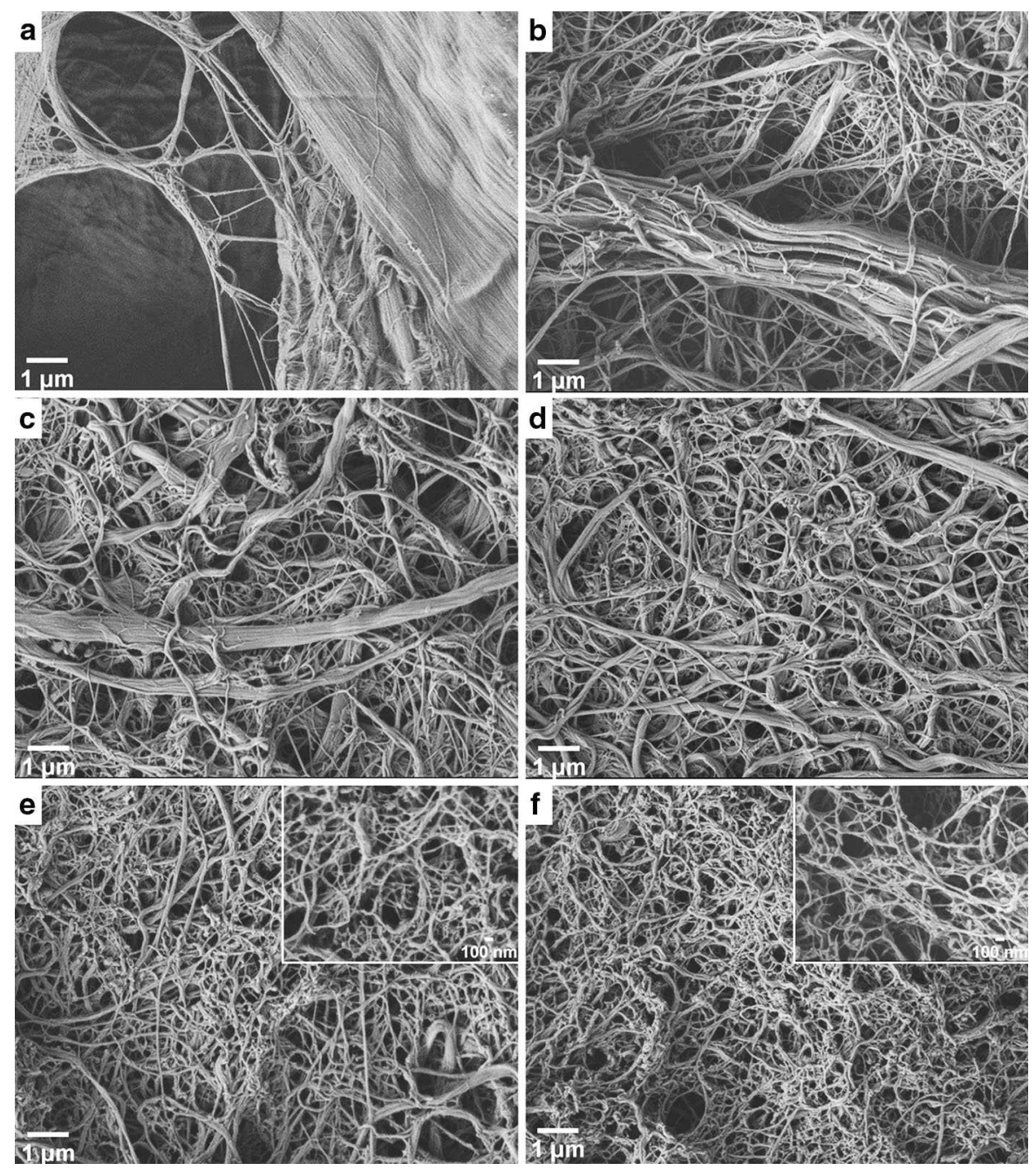

Fig. 1 SEM images of a starting material refined NBKP, b cellulose fibers after 1 pass, c cellulose fibers after 3 passes, d cellulose fibers after 5 passes, e cellulose fibers after 10 passes,

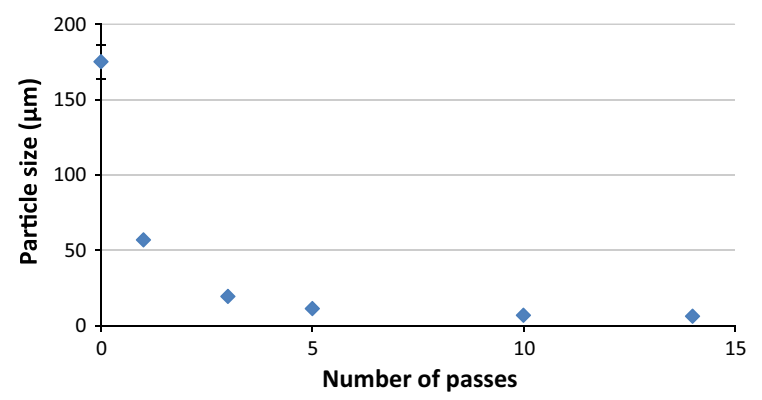

Fig. 2 Particle size of cellulose fibers plotted against the number of passes through a TSE. The particle sizes were evaluated by the laser light scattering method and $\mathbf{f}$ cellulose fibers after 14 passes through a TSE. Insets of (e) and (f): images at higher magnification

size changes could be detected anymore after ten passes through the extruder (see Fig. 1).

However, in laser light scattering, the fibers are taken as spheres; thus, the obtained values should only be taken relatively to each other. In reality, a cellulose fiber does not exist as a single spherical object; moreover, they are entangled and often agglomerated into bigger bundles. Laser light scattering is therefore taken here as a rapid method for the first evaluation of the change in size of cellulose fibers after processing through an extruder. The numbers cannot be taken as absolute values. To our knowledge, the information on the particle size of NFC fibers in suspension evaluated 
by the laser light scattering method has not been documented so far in the literature but is a convenient method to evaluate the degree of fibrillation qualitatively. The particle size obtained after ten passes through a TSE is ca. $6 \mu \mathrm{m}$, which is comparable to spray-dried cellulose nanofibril particles, which are ca. 7-8 $\mu \mathrm{m}$ (Peng et al. 2012).

The regular light transmittance of the composite films at $600-\mathrm{nm}$ wavelength is shown in Fig. 3. The 600 -nm wavelength was chosen as it is in the middle of the visible wavelength range, which is $400-800 \mathrm{~nm}$. A high degree of fibrillation can be indicated by an increase in the transparency of the composite films made from fibrillated cellulose materials. This is because of the generation of nano-size fibrils, which predominantly have diameters below $50 \mathrm{~nm}$ and do not scatter the light (Yano et al. 2005). In general, the higher the number of passes is, the higher the fibrillation effect (compare also Figs. 1, 2); as a result, the composite film becomes more transparent with higher numbers of passes. This indicates that the more uniform size of the fibrils results in enhanced light transmittance of the composites (Iwamoto et al. 2005). The transmittance increases more rapidly, i.e., by $16 \%$, at lower numbers of passes (1-5 passes). After 5 passes up to 14 passes, the transmittance only increases ca. $9 \%$. The highest achieved transmittance is slightly higher than $90 \%$ for cellulose fibers after 14 passes through a TSE, which is very interesting as it is equivalent to that of cellulose nanofibers of $15-\mathrm{nm}$

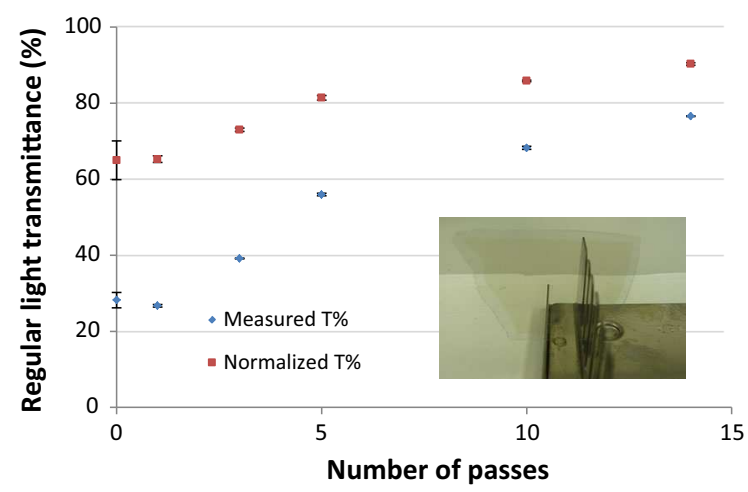

Fig. 3 Regular light transmittance (T\%) of composite films made from acrylic resin and cellulose fibers after $0,1,3,5,10$, and 14 passes through a TSE. The measured T\% values of all films were normalized for a film with $50-\mu \mathrm{m}$ thickness. Inset: Photograph of a composite film made of acrylic resin and cellulose fibers after 14 passes through a TSE. The film is leaned against a supporting metal frame prior to the regular light transmittance measurements width from treated wood powder obtained from a grinder (Abe et al. 2007) or softwood TEMPOoxidized microfbrillated cellulose films (Fukuzumi et al. 2009). These nanocomposite films (Abe et al. 2007; Fukuzumi et al. 2009) also have regular light transmittance of ca. $90 \%$.

The dewatering speed results in Fig. 4 show that the extrusion process definitely has a strong effect on the fibrillation of cellulose fibers. After several passes through the TSE, the dewatering time increases because the aspect ratio and specific surface area of cellulose fibers increase, which subsequently multiplies the number of exposed hydroxyl groups inside the fiber cell walls (Turbak et al. 1983). A higher number of passes results in a better fibrillation effect. The fibers get much smaller and more homogeneous after reaching 10 or 14 passes, respectively (Fig. 2).

However, the decrease in dewatering speed could also indicate an increase in the amorphous portion of the cellulose fibers. The more amorphous the cellulose fibers are, the greater the ability to retain water (Gardner et al. 2008; Iwamoto et al. 2008). The amorphous portion in cellulose generally increases upon degradation (Ho et al. 2011).

Photographs of cellulose fiber settlement after three different time periods are shown in Fig. 5. The sedimentation of cellulose fibers decreases with the increase of the number of passes through the TSE. After $48 \mathrm{~h}$, all cellulose material had sedimented for one- and three-pass samples.

The 5-, 10-, and 14-pass samples are much more stable in aqueous suspension than the others. This indicates that the cellulose fibers of these samples are more homogeneous, smaller in diameter, and larger in surface. This tendency was confirmed by Tingaut et al. (2011) with the observation that a stable suspension of

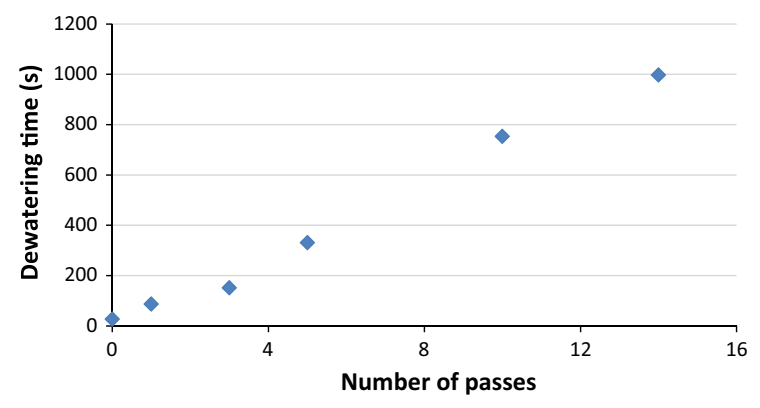

Fig. 4 Dewatering speed of cellulose fibers as a function of the number of passes through a TSE 

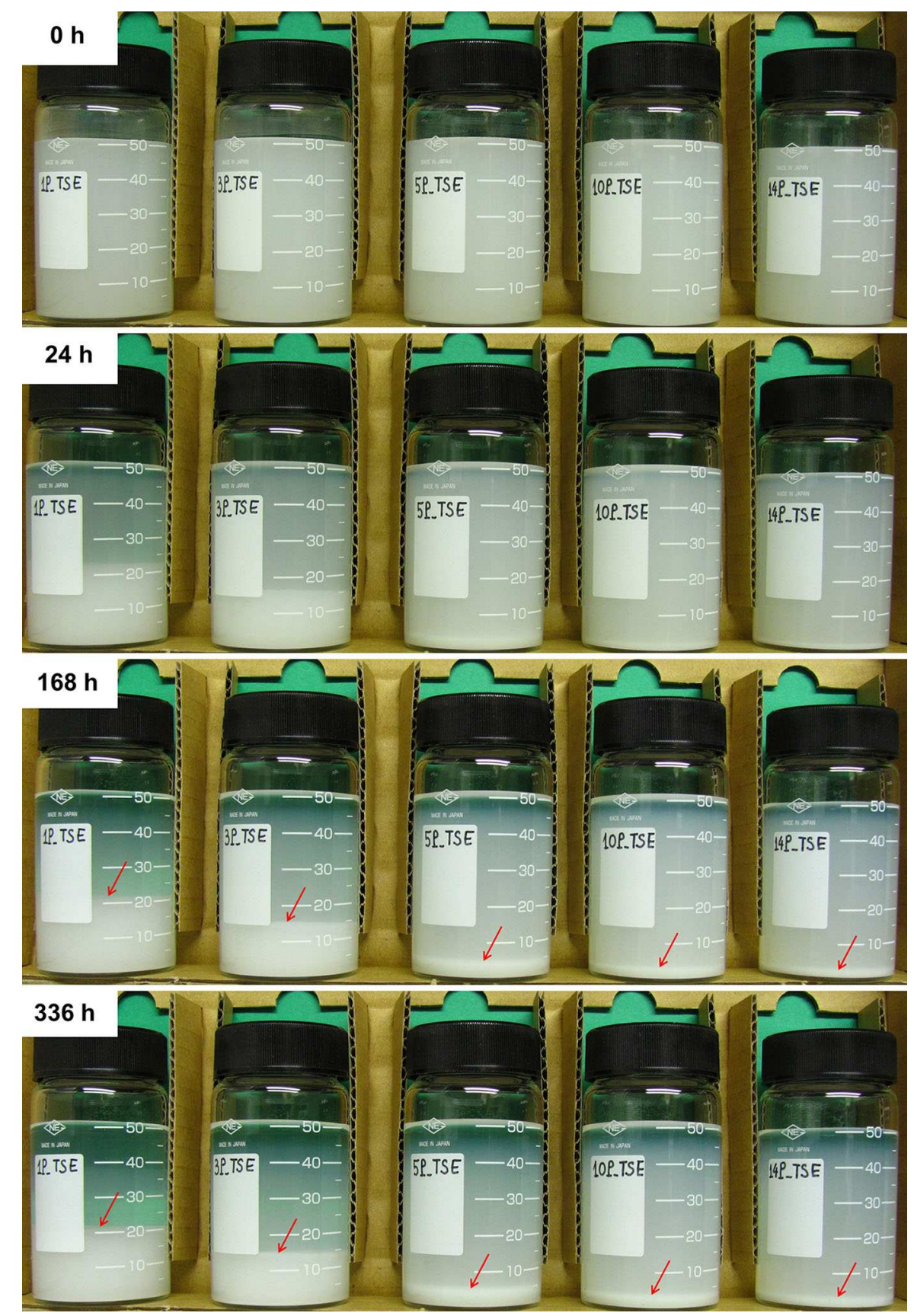

Fig. 5 Photographs after 0, 24, 168 h (1 week), and 336 h (2 weeks) of aqueous suspensions of 0.2 wt $\%$ fibrillated cellulose with various passes through a TSE

carboxymethylated fibrillated cellulose could be obtained for nanofibers of diameters below $100 \mathrm{~nm}$. With 10-15 times passing through the high-shear homogenizer, the aspect ratio of cellulose fibers increased, while further passes (e.g., 20 times) caused the agglomeration of fibrils due to the increase of surface area (Lee et al. 2009b) and surface hydroxyl groups (Zimmermann et al. 2004).

In general, there are two sedimentation levels (Fig. 5). The upper level is formed by finer cellulose 
fibers, which disperse in water even after 2 weeks. The arrows in Fig. 5 indicate the lower sedimentation level, i.e., the sedimentation of bigger fibers or fibril aggregates. In the 5-, 10-, and 14-pass samples, this level is much lower than in the 1- and 3-pass samples. This means that big fibers or fibril aggregates are less present in these samples compared to one- and threepass samples. Figure 5 also indicates that after 1 week, the sedimentation levels are stable.

Effect of the number of passes through a TSE on the degree of degradation of cellulose fibers

The degree of degradation of cellulose fibers was assessed by the results obtained from TGA, XRD, viscosity measurements (used to calculate the degree of polymerization), and tensile tests.

Figure 6 shows the temperature of degradation (DT) obtained from TG analysis of all processed cellulose fibers. The measurements were carried out in nitrogen atmosphere up to $600{ }^{\circ} \mathrm{C}$. Cellulose nanofibers have a thermal degradation around $309{ }^{\circ} \mathrm{C}$ (Jandura et al. 2000). The TGA results show that the extruding process definitely has a strong effect on the degradation of the cellulose fibers. The higher the number of passes is, the lower the degradation temperature. A lower degradation temperature might be associated with the significant decrease in crystallinity (see Fig. 7) observed for the samples (Freire et al. 2006). The fibers become degraded, most prominently after 14 passes through the TSE where the degradation temperature is below $300{ }^{\circ} \mathrm{C}$. However, the degradation temperature is still higher than normal polymer processing temperatures in industry, for example, at $200{ }^{\circ} \mathrm{C}$ for polylactic acid (Jonoobi

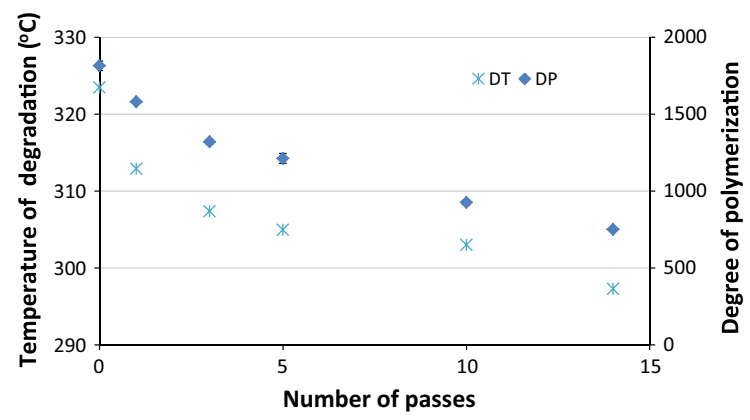

Fig. 6 Degradation temperature (DT) and degree of polymerization (DP) of cellulose fibers as a function of the number of passes through a TSE et al. 2010, 2012) and below $180{ }^{\circ} \mathrm{C}$ for polyethylene and polypropylene (Caulfield et al. 2001).

The DP results (also displayed in Fig. 6) show the same trend as the degradation temperatures (TGA results). The polymerization degree is related to the cellulose chain lengths and therefore provides information on the level of cleavage along the fiber direction. The decrease in polymerization degree therefore indicates a reduction in fiber length after each pass through the extruder. This result is in agreement with the reported DP of cellulose fibers with other disintegration methods. Herrick et al. (1983) observed up to $27 \%$ reduction in the degree of polymerization due to a homogenization process. Mechanical isolation of nanofibers through a highpressure slit homogenizer resulted in an approximately 30-50\% decrease in DP (Henriksson et al. 2007). Iwamoto et al. (2007) reported that after nine passes through a grinder, the DP of nanocellulose changed from ca. 770 to 525 .

The resulting crystallinity ratios calculated from XRD patterns of raw materials and the materials after passing through the extruder $1,3,5,10$, and 14 times are shown in Fig. 7. With up to ten passes, the crystallinity degree of fibrillated cellulose is above $75 \%$. After 14 passes, the degree of crystallinity is lower than $75 \%$. There is no significant change in the crystallinity ratios between one- and five-pass samples. A more distinct decrease (ca. $5 \%$ ) appears after ten passes.

These results suggest that the impact of fibrillation using a TSE on the crystallinity of the cellulose fibers is not significant, especially up to five passes $(<5 \%)$. Iwamoto et al. (2007) obtained a significant decrease in the degree of crystallinity (more than $20 \%$ ) for

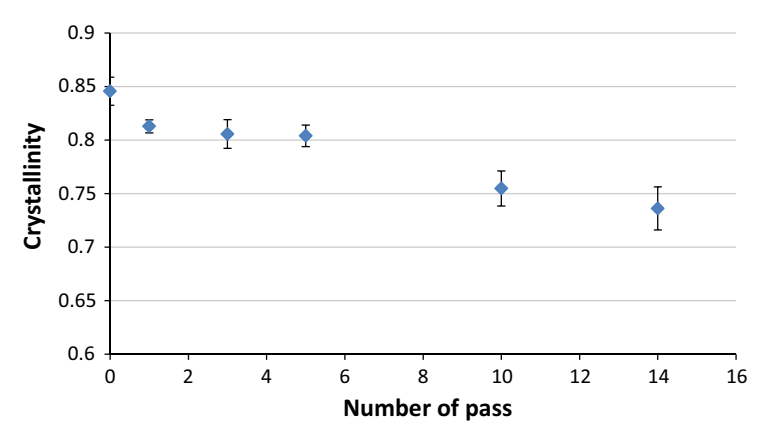

Fig. 7 Degree of crystallinity of the cellulose fibers as a function of the number of passes through a TSE 
increasing the number of passes from 0 to 30 through a grinder. In this case, after five passes through the grinder, the crystallinity of fibrillated pulp fibers decreased more than $15 \%$ compared to non-fibrillated pulp fibers.

Figure 8 shows the mechanical properties of sheet samples (non-fibrillated and fibrillated pulp fibers). When the fibrillation degree increases, the number of hydrogen bonds between available - $\mathrm{OH}$ groups of the fibrillated fibers also increases; therefore, the E-modulus, strength, and strain at break are expected to be higher. This is true for the pulp samples that were passed up to five times through the extruder. However, for 10- and 14-pass samples, the severe treatment (with repeated kneading of the cellulose fibers) caused degradation and in consequence the properties decreased again. The degradation and damage of the fiber (e.g., decrease in the crystallinity, the degradation temperature, and polymerization degree) are demonstrated by previously reported results and support the findings here. The E-modulus is slightly higher after 10 passes and 14 passes compared with other samples, but the strength and strain at break decrease considerably, especially when compared with the 1-, 3-, and 5-pass samples. This higher strength of fibrillated cellulose fiber sheets compared to non-fibrillated ones was related to the higher

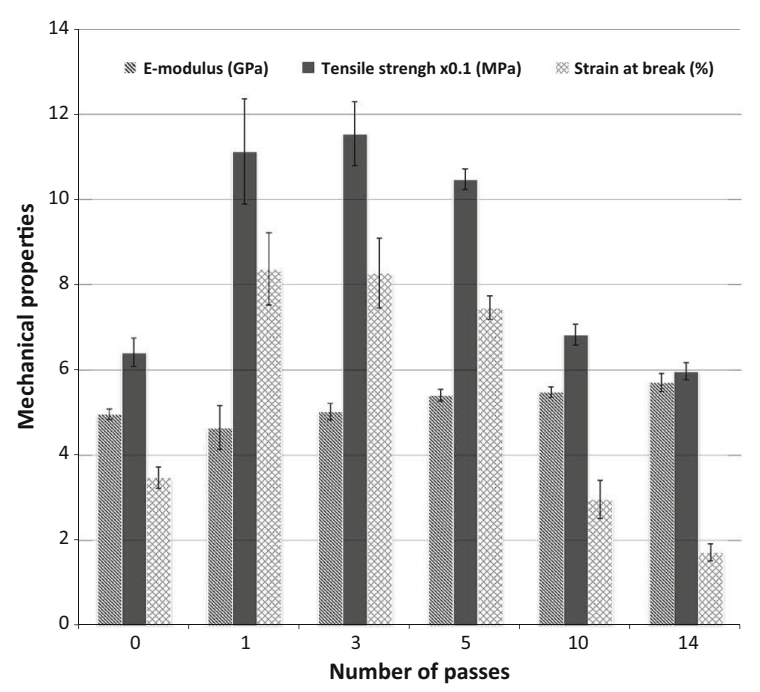

Fig. 8 Tensile properties (E-modulus, tensile strength, and strain at break) of all-cellulose sheets of different passes through a TSE interfibril adhesion properties, greater fibril strength, and smaller and more homogeneously distributed defects (Henriksson et al. 2008). The decrease in polymerization degree contributes to the brittleness of the fibers (refer to previous sections). For the fibrillated cellulose films, it has been proved that the tensile strength increases with higher DP (Henriksson et al. 2007). Therefore, with the lower DP at a higher number of passes, the tensile strength of the fibrillated cellulose sheets displays a decreasing tendency. In general, the mechanical properties are still good after five passes. Further passes through a TSE damage the fibers. Most interestingly from a practical point of view, one-, three-, and five-pass samples show the best mechanical properties. For the reinforcement of thermoplastic resin using a melting compound, the nanofibers are subjected to high temperature and shearing stress (Suzuki et al. 2013, 2014) during melting of the compound, which accelerates deterioration of cellulose nanofibers and reduces reinforcement efficiency.

\section{Degree of fibrillation and degradation}

The analysis results of cellulose fibers were normalized and scaled to the same range, i.e., the maximum value equals 1 and minimum value equals 0 . These were plotted against the number of passes through a TSE (Fig. 9). In this figure, the solid lines represent the fibrillation degree (particle size, light transmittance, and dewatering time), while the dashed lines show the degree of degradation (degradation temperature, DP, crystallinity, and mechanical properties). In summary, the higher the number of passes of cellulose fibers through a TSE, the higher are the fibrillation effect and degradation degree. The fibrillation effect can be proved by the decrease in particle size of cellulose fibers or by the increase in dewatering time of aqueous cellulose suspensions. Particularly, the more fibrillated the fibers are, the higher the light transmittance or transparency of cellulose/acrylic resin composite films. Contrarily, the degradation of cellulose fibers resulted in a decrease in the degradation temperature, degree of polymerization, crystallinity, and mechanical properties of the cellulose materials. In Fig. 9, the intersections between solid and dashed lines indicate the best compromises between the fibrillation and degradation effects of the extrusion process. Such points are highlighted by the arrows (see Fig. 9). Note 
Fig. 9 Degree of fibrillation and degree of degradation of the cellulose fibers as the function of the number of passes through the TSE

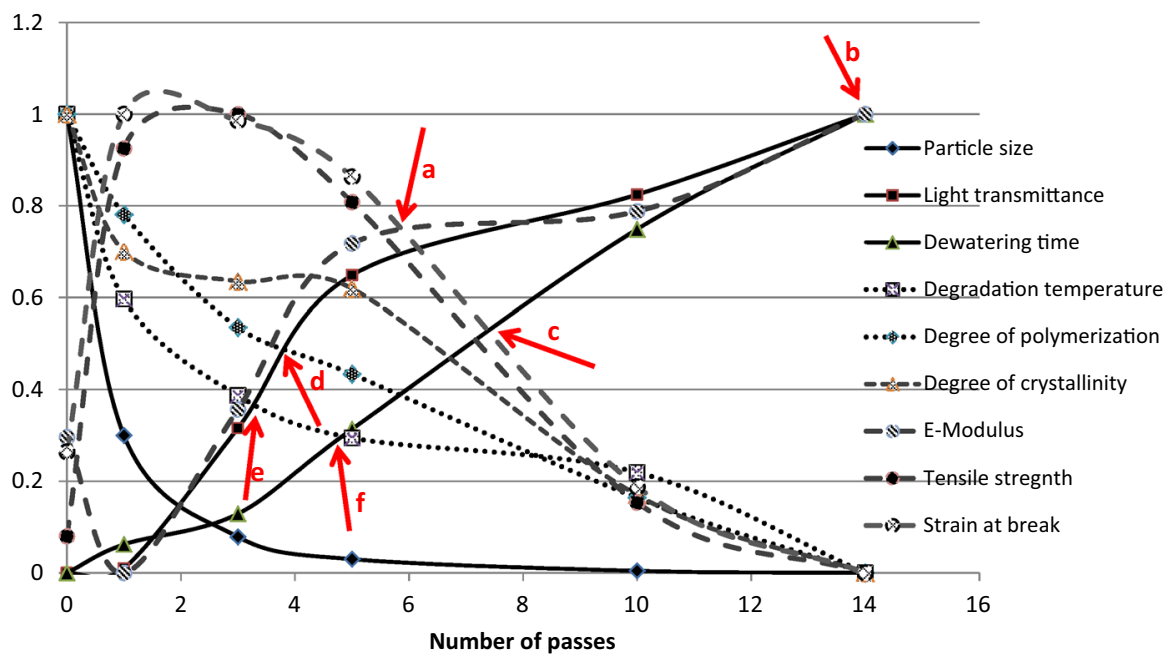

that the results obtained in this study are not general but only for never-dried refined NBKP. Depending on the application purposes, 5-6 passes through a TSE can be used to obtain cellulose nanofibers of the highest possible transparency (highest light transmittance) and crystallinity and the best mechanical properties (see arrow a). Such fibrillated cellulose fibers can find applications in the packaging and electronics sectors. After five passes, the fibrillated cellulose fibers also show the best compromise of dewatering time and degradation temperature (arrow f). Similarly, the highest transparency, longest dewatering speed, but highest possible E-modulus of cellulose fibers could result after 14 passes through a TSE (shown by arrow b).

\section{Conclusions}

Twin-screw extrusion allows the processing and fibrillation of cellulose fibers at high solid contents, which have not been obtained yet with any other method described in the literature so far. Thus, $28 \mathrm{wt} \%$ solid content kraft pulp could be disintegrated to high-quality fibrillated cellulose fibers of ca. $33-45$ wt\% solid content. For practical reasons, high solid-content fibrillated cellulose processed with the introduced new fibrillation method will be of great interest for industry. With high-solid-content materials, less water has to be transported. A powder form of fibrillated cellulose as obtained after extrusion is necessary for its utilization in industry, especially in the field of thermoplastic processing, as nanocomposites could be produced much more easily. In addition, extrusion is already a common method used in many industries.

However, the fibrillation of fibers was only possible up to 14 passes through the extruder in this study because of heat generation. Not only fibrillation but also degradation occurred to a certain degree in cellulose fibers after several consecutive passes through a TSE. These effects were studied and evaluated by various characterization methods. For never-dried refined NBKP, it can be concluded that the best compromise between the fibrillation degree and acceptable degradation appeared at 3, 4, 5, 8, and 14 passes. The right number of passes should be selected depending on the quality requirement for the envisaged application of the cellulose fibers.

Acknowledgments We kindly acknowledge the Mission Research Program for a Sustainable Humanosphere, RISH, Kyoto University, for financial support. We thank Esther Strub from EMPA for performing the viscosity measurement. We are very grateful to Prof. Yano's laboratory members for their useful advice and support.

\section{References}

Abe K, Iwamoto S, Yano H (2007) Obtaining cellulose nanofibers with a uniform width of $15 \mathrm{~nm}$ from wood. Biomacromolecules 8:3276-3278. doi:10.1021/bm700624p

Arthur NW, Anthony JR (1998) Polymer processing and structure development. Kluwer, Dordrecht

Caulfield DF, Jacobson RE, Sears KD, Underwood JH (2001) Woodpulp fibres as reinforcements for high-melting 
engineering thermoplastics for "under-the-hood" automotive applications. In: Polymer processing society 17 th annual meeting, Montreal, Canada

Dufresne A (2012) Nanocellulose: from nature to high performance tailored materials. Walter De Gruyter, Berlin

Dufresne A (2013) Nanocellulose: a new ageless bionanomaterial. Mater Today 16:220-227. doi:10.1016/j.mattod. 2013.06.004

Eyholzer C, Bordeanu N, Lopez-Suevos F, Rentsch D, Zimmermann T, Oksman K (2010) Preparation and characterization of water-redispersible nanofibrillated cellulose in powder form. Cellulose 17:19-30

Freire CSR, Silvestre AJD, Neto CP, Belgacem MN, Gandini A (2006) Controlled heterogeneous modification of cellulose fibers with fatty acids: effect of reaction conditions on the extent of esterification and fiber properties. J Appl Polym Sci 100:1093-1102. doi:10.1002/app.23454

Fukuzumi H, Saito T, Iwata T, Kumamoto Y, Isogai A (2009) Transparent and high gas barrier films of cellulose nanofibers prepared by TEMPO-mediated oxidation. Biomacromolecules 10:162-165. doi:10.1021/bm801065u

Gardner DJ, Oporto GS, Mills R, Samir MASA (2008) Adhesion and surface issues in cellulose and nanocellulose. J Adhes Sci Technol 22:545-567. doi:10.1163/156856108X295509

Gruber E, Gruber R (1981) Viscosimetrical determination of the degree of polymerization of cellulose. Papier 35:133-141

Henriksson M, Henriksson G, Berglund LA, Lindström T (2007) An environmentally friendly method for enzyme-assisted preparation of microfibrillated cellulose (MFC) nanofibers. Eur Polym J 43:3434-3441

Henriksson M, Berglund LA, Isaksson P, Lindstrom T, Nishino T (2008) Cellulose nanopaper structures of high toughness. Biomacromolecules 9:1579-1585. doi:10.1021/bm800038n

Herrick FW, Casebier RL, Hamilton JK, Sandberg KR (1983) Microfibrillated cellulose: morphology and accessibility. J Appl Polym Sci: Appl Polym Symp 37:797-813

Hietala M, Niinimäki J, Oksman K (2011a) The use of twin-screw extrusion in processing of wood: the effect of processing parameter and pretreatment. BioResources 6:4615-4625

Hietala M, Samuelsson E, Niinimäki J, Oksman K (2011b) The effect of pre-softened wood chips on wood fibre aspect ratio and mechanical properties of wood-polymer composites. Compos Part A 42:2110-2116. doi:10.1016/j. compositesa.2011.09.021

Hietala M, Rollo P, Kekäläinen K, Oksman K (2014) Extrusion processing of green biocomposites: compounding, fibrillation efficiency, and fiber dispersion. J Appl Polym Sci 131. doi:10.1002/app.39981

Ho T, Zimmermann T, Hauert R, Caseri W (2011) Preparation and characterization of cationic nanofibrillated cellulose from etherification and high-shear disintegration processes. Cellulose 18:1391-1406. doi:10.1007/s10570-011-9591-2

Hubbe MA, Rojas OJ, Lucia LA, Sain M (2008) Cellulosic nanocomposites: a review. BioResources 3:929-980

Hult EL, Larsson PT, Iversen T (2001) Cellulose fibril aggregation-an inherent property of kraft pulps. Polymer 42:3309-3314. doi:10.1016/S0032-3861(00)00774-6

Iwamoto S, Nakagaito AN, Yano H, Nogi M (2005) Optically transparent composites reinforced with plant fiber-based nanofibers. Appl Phys A Mater Sci Process 81:1109-1112
Iwamoto S, Nakagaito AN, Yano H (2007) Nano-fibrillation of pulp fibers for the processing of transparent nanocomposites. Appl Phys A Mater Sci Process 89:461-466

Iwamoto S, Abe K, Yano H (2008) The effect of hemicelluloses on wood pulp nanofibrillation and nanofiber network characteristics. Biomacromolecules 9:1022-1026. doi:10. 1021/bm701157n

Jandura P, Riedl B, Kokta BV (2000) Thermal degradation behavior of cellulose fibers partially esterified with some long chain organic acids. Polym Degrad Stab 70:387-394. doi:10.1016/S0141-3910(00)00132-4

Jonoobi M, Harun J, Mathew AP, Oksman K (2010) Mechanical properties of cellulose nanofiber (CNF) reinforced polylactic acid (PLA) prepared by twin screw extrusion. Compos Sci Technol 70:1742-1747. doi:10.1016/j. compscitech.2010.07.005

Jonoobi M, Mathew A, Abdi M, Makinejad M, Oksman K (2012) A comparison of modified and unmodified cellulose nanofiber reinforced polylactic acid (PLA) prepared by twin screw extrusion. J Polym Environ 20:991-997. doi:10. 1007/s10924-012-0503-9

Josset S, Orsolini P, Siqueira G, Tejado A, Tingaut P, Zimmermann T (2014) Energy consumption of the nanofibrillation of bleached pulp, wheat straw and recycled newspaper through a grinding process. Nord Pulp Pap Res J 29:167-175. doi:10.3183/NPPRJ-2014-29-01-p167-175

Lee S-H, Teramoto Y, Endo T (2009a) Enzymatic saccharification of woody biomass micro/nanofibrillated by continuous extrusion process I- effect of additives with cellulose affinity. Bioresour Technol 100:275-279. doi:10.1016/j. biortech.2008.05.051

Lee S-Y, Chun S-J, Kang I-A, Park J-Y (2009b) Preparation of cellulose nanofibrils by high-pressure homogenizer and cellulose-based composite films. J Ind Eng Chem 15:5055. doi:10.1016/j.jiec.2008.07.008

Lee S-H, Inoue S, Teramoto Y, Endo T (2010) Enzymatic saccharification of woody biomass micro/nanofibrillated by continuous extrusion process II: effect of hot-compressed water treatment. Bioresour Technol 101:9645-9649. doi:10.1016/j.biortech.2010.07.068

Lin Z, Liu L, Li R, Shi J (2012) Screw extrusion pretreatments to enhance the hydrolysis of lignocellulosic biomass. J Microb Biochem Technol S12

Liu C, van der Heide E, Wang H, Li B, Yu G, Mu X (2013) Alkaline twin-screw extrusion pretreatment for fermentable sugar production. Biotechnol Biofuels 6:97

Paakko M et al (2007) Enzymatic hydrolysis combined with mechanical shearing and high-pressure homogenization for nanoscale cellulose fibrils and strong gels. Biomacromolecules 8:1934-1941. doi:10.1021/bm061215p

Peng Y, Gardner D, Han Y (2012) Drying cellulose nanofibrils: in search of a suitable method. Cellulose 19:91-102. doi:10.1007/s10570-011-9630-z

Portnoy RC (1998) Medical plastics: degradation resistance and failure analysis. Norwich, NY: Society of Plastics Engineers [etc.], Norwich, NY

Segal L, Creely JJ, Martin AE, Conrad CM (1959) An empirical method for estimating the degree of crystallinity of native cellulose using the X-ray diffractometer. Text Res J 29:786-794. doi:10.1177/004051755902901003 
Siró I, Plackett D (2010) Microfibrillated cellulose and new nanocomposite materials: a review. Cellulose 17:459-494. doi:10.1007/s10570-010-9405-y

Suzuki K, Okumura H, Kitagawa K, Sato S, Nakagaito A, Yano H (2013) Development of continuous process enabling nanofibrillation of pulp and melt compounding. Cellulose 20:201-210. doi:10.1007/s10570-012-9843-9

Suzuki K, Sato A, Okumura H, Hashimoto T, Nakagaito A, Yano H (2014) Novel high-strength, micro fibrillated cellulose-reinforced polypropylene composites using a cationic polymer as compatibilizer. Cellulose 21:507-518. doi:10.1007/s10570-013-0143-9

Thygesen A, Oddershede J, Lilholt H, Thomsen AB, Ståhl K (2005) On the determination of crystallinity and cellulose content in plant fibres. Cellulose 12:563-576. doi:10.1007/ s10570-005-9001-8

Tingaut P, Eyholzer C, Zimmermann T (2011) Functional polymer nanocomposite materials from microfibrillated cellulose. In: Hashim A (ed) Advances in nanocomposite technology. InTech. doi:10.5772/20817

Turbak AF, Snyder FW, Sandberg KR (1983) Microfibrillated cellulose, a new cellulose product: properties, uses, and commercial potential. J Appl Polym Sci: Appl Polym Symp 37:815-827

White JL (1991) Twin screw extrusion: technology and principles. Hanser Publishers, Munich

Yano H, Sugiyama J, Nakagaito AN, Nogi M, Matsuura T, Hikita M, Handa K (2005) Optically transparent composites reinforced with networks of bacterial nanofibers. Adv Mater 17:153-155. doi:10.1002/adma.200400597

Yano H, Yano K, Mogi Y (2007) Method for producing aliphatic polyester composition, pulp used for the same, and cellulosic fiber, and method for microfibrillating the same. Japan Patent 4127316

Zimmermann T, Pöhler E, Geiger T (2004) Cellulose fibrils for polymer reinforcement. Adv Eng Mater 6:754-761

Zimmermann T, Pöhler E, Schwaller P (2005) Mechanical and morphological properties of cellulose fibril reinforced nanocomposites. Adv Eng Mater 7:1156-1161

Zimmermann T, Bordeanu N, Strub E (2010) Properties of nanofibrillated cellulose from different raw materials and its reinforcement potential. Carbohydr Polym 79:1086-1093 